International Journal of Wireless \& Mobile Networks (IJWMN) Vol. 3, No. 2, April 2011

\title{
Enhancing The Performance of THE DNDP ALGORITHM
}

\author{
Hussein Al-Bahadili ${ }^{1}$, Ghassan Issa $^{1}$, Alia Sabri $^{2}$ \\ ${ }^{1}$ Faculty of Information Technology, Petra University, Jordan \\ ${ }^{2}$ Faculty of Information Technology, Applied Science University, Jordan \\ hbahadili@uop.edu.jo, gissa@uop.edu.jo, a_sabri@asu.edu.jo
}

\begin{abstract}
A novel dynamic noise-dependent probabilistic (DNDP) route discovery algorithm was recently developed to enhance the performance of the dynamic probabilistic algorithm in noisy mobile ad hoc networks (MANETs). In this algorithm, the mathematical model for calculating node retransmission probability $\left(p_{t}\right)$ is calculated as a function of two independent variables: number of first-hop neighbors $(k)$ and probability of reception $\left(p_{c}\right)$. The model also shows another independent variable, namely, the maximum retransmission probability that can be assigned to the transmitting node $\left(p_{t, p c m i n}\right)$, which is assumed to be a fixed value. In this paper, we propose a new mathematical model for calculating $p_{t}$. In this new model $p_{t, p c m i n}$ is calculated as a function of $k$. The performance of the DNDP algorithm using fixed and $k$-dependent $p_{t, p c m i n}$ is evaluated through simulations. The simulation results showed that the new model enhances the performance of the DNDP algorithm as it significantly reduces the number of retransmissions at an insignificant reduction in the network reachability.
\end{abstract}

\section{KEYWORDS}

MANETs, dynamic routing protocols, route discovery, flooding optimization algorithms, probabilistic algorithm, the DNDP algorithm.

\section{INTRODUCTION}

A mobile ad hoc network (MANET) is defined as a collection of low-power wireless mobile nodes forming a temporary network without the aid of any established infrastructure or centralized administration [1,2]. A data packet in MANET is forwarded to other mobile nodes on the network through reliable and efficient routing protocols, which are usually implemented in software as part of the network layer [3]. Dynamic routing protocols (DRPs), such as: ad hoc on-demand distance vector routing (AODV) [4], dynamic source routing (DSR) [5], zone routing protocol (ZRP) [6], and location-aided routing (LAR) [7], are widely used routing protocols in MANETs.

The DRPs consist of two phases: (i) route discovery, which is initiated when a node (source) desires to send a data packet to some node (destination) and does not have a valid route to that destination, and (ii) route maintenance, which is periodically initiated to maintain existing route; and if the route is broken for any reason, then the source either finds and uses other recognized route on its routing table or if it cannot find a route, it initiates a new route discovery procedure [3]. It is well approved that the cost of information exchange during route discovery is higher than the cost of point-to-point data forwarding after the route is discovered. Thus, significant efforts have been put forward by researchers to minimize the cost of route discovery [8].

In route discovery, the source initiates and broadcasts to its neighbours a small packet called route request packet (RREQ), which carries information on the source and destination addresses, packet sequence number (ID), and specific lifetime, i.e., time-to-live (TTL). Each recipient node checks to see if it is the destination; if not, then it appends its address to the payload of the RREQ and forwards it to its neighbours, and so on until the expiration of the

DOI : 10.5121/ijwmn.2011.3210 
International Journal of Wireless \& Mobile Networks (IJWMN) Vol. 3, No. 2, April 2011

RREQ. If the RREQ reaches its destination, the destination sends a route reply packet (RREP) back to the source through the route from which it first received that particular RREQ [3,5]. If the RREQ expires before reaching its destination, the node at which it expires, sends a route error packet (RERR) back to the source, once again through the route from which it first received that particular RREQ. As result of this route discovery failure, the source initiates a new RREQ and continues to do so until a route is found.

Pure flooding is one of the simplest and reliable mechanisms proposed in the literature for route discovery in MANETs $[1,9]$. The main drawback of pure flooding is that it is very costly where it costs $n$ transmissions in a network of $n$ reachable nodes. In addition, it results in serious redundancy, contention, and collisions in the network; such a scenario has often been referred to as the broadcast storm problem (BSP) [10].

To eliminate the effects of BSP, a variety of flooding optimization algorithms have been developed, such as: probabilistic [1, 2, 11], multipoint relaying (MPR) [12, 13], counter/distance/location/cluster -based [3, 7, 10, 14] algorithms. They all try to minimize contention and collisions by limiting the number of retransmissions. As the number of retransmissions is decreased, the bandwidth and power are saved and contentions and collisions are reduced, and consequently the overall network performance is improved.

Probabilistic algorithm has been widely-used for route discovery in MANETs [1, 2]. However, it has been demonstrated that the performance of the probabilistic algorithm is severely suffered in presence of noise due to increases packet-loss (data or RREQ packets), and consequently reduces the overall network performance $[15,16]$.

A dynamic noise-dependent probabilistic (DNDP) algorithm was proposed by Al-Bahadili and Sabri in [17] for route discovery in noisy MANETs. In this algorithm, the retransmission probability of the transmitting node $\left(p_{t}\right)$ is modeled as a function of two independent variable; these are: the number of first-hop neighbors $(k)$ and the probability of reception $\left(p_{c}\right)$. The model also shows another independent variable, namely, the maximum retransmission probability that can be assigned to the transmitting node $\left(p_{t, p c m i n}\right)$, which is assumed to be a fixed value.

In this paper, we propose a new mathematical model for calculating $p_{t}$. In this new model, $p_{t, p c m i n}$ is calculated as a function of $k$. The new model is implemented on the MANET simulator (MANSim) [18], and the performance of the DNDP algorithm using fixed and $k$-dependent $p_{t, p c m i n}$ is evaluated through simulations. The simulation results showed that the new model enhances the performance of the DNDP algorithm as it reduces the number of retransmissions at an insignificant reduction in the network reachability.

This section provides an introduction to the general domain of this paper. The rest of the paper is organized as follows. Related work is discussed in Section 2. The dynamic probabilistic and the DNDP algorithms are discussed in Sections 3 and 4, respectively. The proposed model is described in Section 5. Simulation results are presented and discussed in Section 6. Finally, in Section 7, conclusions are drawn and recommendations for future work are pointed-out.

\section{Previous Work}

This section reviews some of the most recent and related work on probabilistic flooding in both noiseless and noisy MANETs. Probabilistic algorithm was first used by Haas et. al. [19] for route discovery in ad hoc networks, and they called it a gossip-based route discovery (GOSSIP1) approach. They used a predefined $p_{t}$ to decide whether or not a node forwards the RREQ packets. GOSSIP1 has a slight problem with initial conditions. If the source has relatively few neighbors, there is a chance that none of them will gossip, and the gossip will die. To make sure this does not happen, Haas et. al. later developed a modified protocol, in which they gossip with $p_{t}=1$ for the first $h$ hops before continuing to gossip with $p_{t}<1$. Their results showed that they can save up to $35 \%$ message overhead compared to simple flooding. 
S. Tseng et. al. [10] investigated the performance of the probabilistic flooding for various network densities in noise-free environment. They presented results for three network parameters, namely, reachability, saved rebroadcast, and average latency, as a function of $p_{t}$ and network density. Sasson et. al. [20] suggested exploring algorithms in which nodes would dynamically adjust their $p_{t}$ based on local topology information. Because in their work they made the assumption that all nodes possess the same transmission range, they suggested another potential area for study which is to modify $p_{t}$ according to the node radio transmission range.

Kim et. al. [21] introduced a broadcasting scheme in which a node dynamically adjusts its $p_{t}$ according to its additional coverage area. The additional coverage is estimated by the distance from the sender. The simulation results showed this scheme generates fewer rebroadcasts than pure flooding approach. It also incurs lower broadcast collision without sacrificing high reachability.

Scott and Yasinsac [2] presented a dynamic probabilistic solution that is appropriate to solving BSPs in dense mobile networks. The approach can prevent broadcast storms during flooding in dense networks and can enhance comprehensive delivery in sparse networks.

Barret et. al. [22] introduced a probabilistic routing protocol for sensor networks. In this protocol, a sensor decides to forward a message with $p_{t}$ that depends on various parameters, such as the distance of the sensor to the destination, the distance of the source sensor to the destination, or the number of hops a packet has already traveled. They proposed two protocol variants and compared the new methods to other probabilistic and deterministic protocols. The results showed that the multi-path protocols are less sensitive to misinformation, and suggest that in the presence of noisy data, a limited flooding strategy will actually perform better and use fewer resources than an attempted single-path routing strategy, also parametric probabilistic protocols outperforms other protocols. The results also suggested that protocols using network information perform better than protocols that do not, even in the presence of strong noise.

Viswanath and Obraczka [23] developed an analytical model to study the performance of plain and probabilistic flooding in terms of its reliability and reachability in delivering packets. Their results indicated that probabilistic flooding can provide similar reliability and reachability guarantees as plain flooding at a lower overhead.

Zhang and Agrawal [24] proposed a probabilistic scheme that dynamically adjusts $p_{t}$ as per node distribution and node movement. The scheme combines between probabilistic and counterbased approaches. They evaluated the performance of their scheme by comparing it with simple flooding and fixed probabilistic algorithms. Simulation results showed that the new scheme performs better than the two algorithms.

Abdulai et. al. [25] studies the performance of the AODV protocol over a range of possible $p_{t}$. They focused on the route discovery part of the routing algorithm, they modified the AODV implementation to incorporate $p_{t}$; the RREQ packets are forwarded in accordance with a predetermined $p_{t}$. Simulation results showed that setting efficient $p_{t}$ has a significant effect on the general performance of the protocol. The results also revealed that the optimal $p_{t}$ for efficient performance is affected by the prevailing network conditions such as traffic load, node density, and node mobility. During their study they observed that the optimal $p_{t}$ is around 0.5 in the presence of dense network conditions and around 0.6 for sparse network conditions.

Bani-Yassein et. al. [1] proposed a dynamic probabilistic flooding algorithm in MANETs to improve network reachability and saved rebroadcast. The algorithm determines $p_{t}$ by considering the network density and node movement. This is done based on locally available information and without requiring any assistance of distance measurements or exact location determination devices. The algorithm controls the frequency of rebroadcasts and thus might save network resources without affecting delivery ratios. 
Abdulai et. al. [26] proposed two probabilistic methods for on-demand route discovery, that is simple to implement and can significantly reduce the overhead involved in the dissemination of RREQs. The two probabilistic methods are: the adjusted probabilistic (AP) and the enhanced adjusted probabilistic (EAP) which address the broadcast storm problem in the existing OADV routing protocols.

Bani Yassein et. al. [9] combined probabilistic and knowledge based approaches on the AODV protocol to enhance the performance of existing protocol by reducing the communication overhead incurred during the route discovery process. The simulation results revealed that equipping AODV with fixed and adjusted probabilistic flooding helps to reduce the overhead of the route discovery process whilst maintaining comparable performance levels in terms of saved rebroadcasts and reachability as achieved by conventional AODV. Moreover, the results indicated that the adjusted technique results in better performance compared to the fixed one.

Khan et. al. [27] proposed a coverage-based dynamically adjusted probabilistic forwarding scheme and compared its performance with simple and fixed probabilistic schemes. The proposed scheme keeps up the reachability of simple flooding while maintaining the simplicity of probability based schemes.

Hanash et. al. [28] proposed a dynamic probabilistic broadcast approach that can efficiently reduce broadcast redundancy in MANETs. The algorithm dynamically calculates $p_{t}$ according to $k$. They compared their approach against simple flooding approach, fixed probabilistic approach, and adjusted probabilistic flooding by implementing them in a modified version of the AODV protocol using GloMoSim. The simulation results showed that broadcast redundancy can be significantly reduced through their approach while keeping the reachability high.

Al-Bahadili [29] developed a new retransmission probability adjusting model, in which the neighborhood densities are divided into three regions (low, medium, and high). The performance of the new model was evaluated and compared with pure and other probabilistic algorithms. The model enhances the performance of probabilistic broadcast by reducing the number of transmissions while keeping almost the same network reachability.

Al-Bahadili and Kaabned [15] investigated the effect of noise-level on the performance of the probabilistic algorithm in MANETs. They investigated the effect of node density, node average speed, radio transmission range, $p_{t}$, and $p_{c}$ on number of retransmissions, duplicate reception, average hop count, and reachability. Their results showed that the performance of the network is severely suffered as $p_{c}$ increases, i.e. the noise-level increases.

\section{The Dynamic Probabilistic Algorithm}

In probabilistic broadcast, a node retransmits the packet with a certain $p_{t}$ and with probability $\left(1-p_{t}\right)$ it discards the packet $[1,2]$. A node is allowed to retransmit a given RREQ only once, i.e., if a node receives a RREQ, it checks to see if it has retransmitted it before, if so then it just discards it, otherwise it performs its probabilistic retransmission check. Nodes usually can identify the RREQ through its sequence number. The source node $p_{t}$ is always set to 1 , to enable it initializing a RREQ. On other hand, the $p_{t}$ of the intermediate nodes (all nodes except the source and destination) can be set by using one of the following approaches:

(1) Static approach in which a pre-determined $p_{t}$ is set for each node on the network and it can be expressed as: $p_{t}=P_{t}$, where $P_{t}$ is a constant value $\left(0<P_{t} \leq 1\right) . P_{t}=1$ for pure flooding.

(2) Dynamic approach in which each node on the network locally calculates its $p_{t}$ as a function of $k\left(p_{t}(k)\right)$, where $p_{t}(k)$ could be any linear/nonlinear function of $k$. 
In this paper, we mainly concern with dynamic probability, therefore, in the next section, we shall provide a description of an efficient and flexible function that will be used for dynamically calculate $p_{t}(k)$ for intermediate nodes on the network.

\subsection{Calculation of $p_{t}(k)$}

Many functions have been developed for calculating $p_{t}(k)[1-2,19-21,24,29]$. However, in this paper, the discrete function presented in [29] is used for calculating $p_{t}(k)$. This function is shown in Figure 1. It demonstrates a satisfactory performance when used in various network conditions and it is mathematically expressed as:

$$
p_{t}(k)= \begin{cases}p_{\max } & \text { for } k \leq N_{1} \\ p_{1}-\frac{k-N_{1}}{N_{2}-N_{1}}\left(p_{1}-p_{2}\right) & \text { for } N_{1}<k<N_{2} \\ p_{\min } & \text { for } k \geq N_{2}\end{cases}
$$

Where $p_{\min }$ and $p_{\max }$ are the minimum and maximum retransmission probabilities, $N_{l}$ is the number of nodes at or below which $p_{t}(k)=p_{\max }, N_{2}$ is the number of nodes at or above which $p_{t}(k)=p_{\min }$, and $p_{1}$ and $p_{2}$ are the retransmission probabilities assign to intermediate nodes when they have $N_{1}+1$ and $N_{2}-1$ first-hop neighboring nodes. $p_{1}$ and $p_{2}$ should lie between $p_{\max }$ and $p_{\min }$, and also $p_{1}$ is always $\geq p_{2}$.

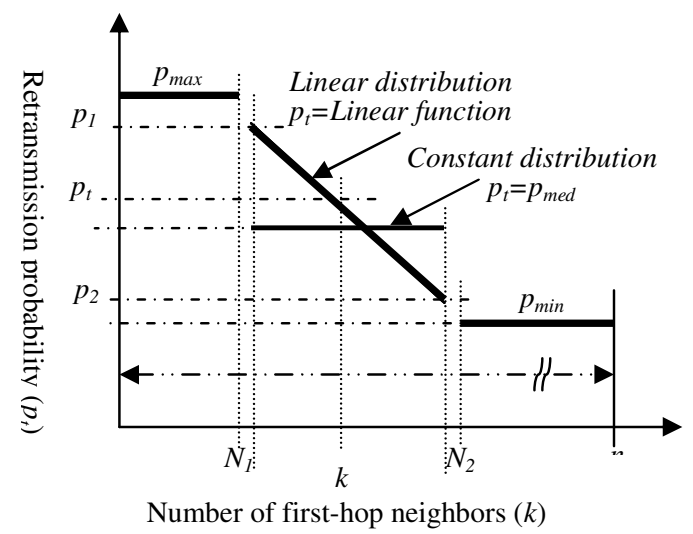

Figure 1. The $k$-dependent retransmission probability $\left(p_{t}(k)\right)$

In general, selection of a satisfactory distribution within the interval $\left[N_{I}+1, N_{2}-1\right]$ and the values of $p_{\max }, p_{\min }, p_{1}, p_{2}, N_{1}$, and $N_{2}$ depend on a number of factors and need to be carefully selected. In this work, the values in [29] are used, which are $0.8,0.5,0.8,0.5,4$, and 15 for $p_{\max }, p_{\min }, p_{1}$, $p_{2}, N_{1}$, and $N_{2}$.

Figure 2 outlines the main phases of the dynamic probabilistic algorithm in a noisy environment. To accommodate the effect of noise, it can be seen in Figure 2 that when the node is within the ratio transmission range of the transmitting node, a test is performed to find-out whether the RREQ packet is successfully delivered or not. This is tested by generating a random number $\xi_{1}$ and comparing it with $p_{c}$. If $\xi_{I} \leq p_{c}$, then the packets is successfully delivered to the receiving node, otherwise, it is undelivered $[15,16]$.

\section{THE DNDP ALgORITHM}

The DNDP algorithm was proposed to enhance the performance of dynamic probabilistic algorithm in noisy MANETs. In this algorithm, instead of calculating $p_{t}$ as a function of $k$ only, $p_{t}$ is determined locally by the retransmitting nodes considering both $k$ and $p_{c}\left(p_{t}\left(k, p_{c}\right)\right)$ as:

$$
p_{t}\left(k, p_{c}\right)=p_{t}(k)+p_{t}\left(p_{c}\right)
$$


International Journal of Wireless \& Mobile Networks (IJWMN) Vol. 3, No. 2, April 2011

Where $p_{t}\left(p_{c}\right)$ is the noise-dependent $p_{t}$.

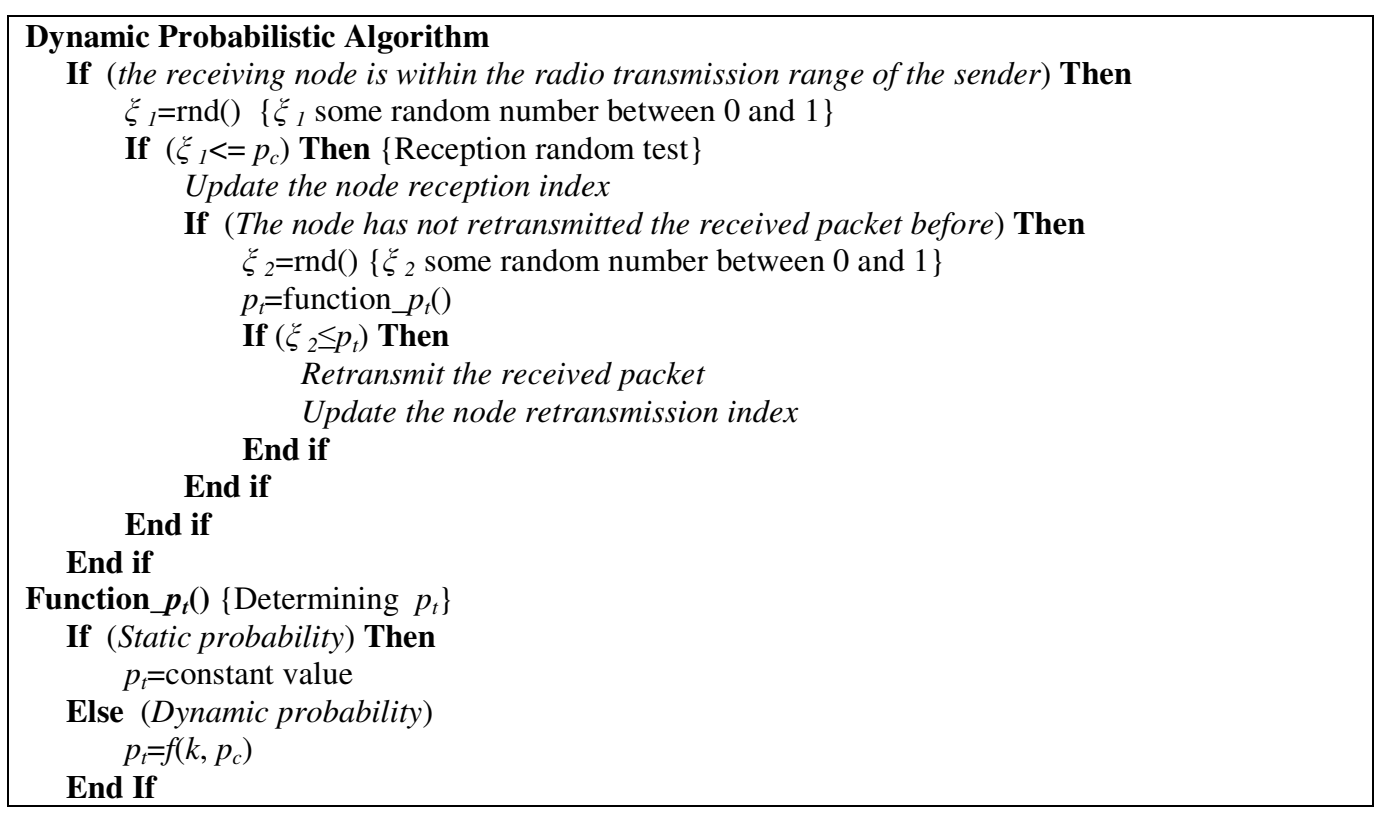

Figure 2. The dynamic probabilistic algorithm

\subsection{Calculation of $p_{t}\left(k, p_{c}\right)$}

It can be seen from Eqn. 2 that in order to calculate $p_{t}\left(k, p_{c}\right)$, we need to calculate $p_{t}(k)$ and $p_{t}\left(p_{c}\right) . p_{t}(k)$ can be calculated using Eqn. 1, and in this section we discuss the calculation of $p_{t}\left(p_{c}\right)$. The following main constraints should be considered when calculating $p_{t}\left(p_{c}\right)$,:

(1) The value of $p_{t}\left(p_{c}\right)$ should be $\geq 0$ and $\leq 1-p_{t}(k)$, so that $p_{t}\left(k, p_{c}\right)$ will always be $\leq 1$.

(2) The value of $p_{t}\left(k, p_{c}\right)$ lies between $p_{t}(k)$ and $p_{t, p c m i n}$, which is a pre-adjusted maximum allowable $p_{t}$ at a certain minimum value of $p_{c}\left(p_{c, m i n}\right)$.

(3) The value of $p_{t, p c m i n}$ should be $\leq 1$ and $\geq p_{t}(k)$.

Considering the above constraints, $p_{t}\left(p_{c}\right)$ can be calculated as:

$$
p_{t}\left(p_{c}\right)=\alpha\left(p_{t, p c m i n}-p_{t}(k)\right)
$$

Where $\alpha$ is called the noise-correction factor, which is a function of $p_{c}$, and has a value that lies between 0 and 1. Substituting Eqn. 3 into Eqn. 2 yields:

$$
p_{t}\left(k, p_{c}\right)=p_{t}(k)+\alpha\left(p_{t, p c m i n}-p_{t}(k)\right)
$$

In [17], the value of $\alpha$ is calculated using the following equation:

$$
\alpha=\frac{1-p_{c}}{1-p_{c, \min }}
$$

Now, substituting Eqn. 5 into Eqn. 4 yields the following general equation for $p_{t}\left(k, p_{c}\right)$ :

$$
p_{t}\left(k, p_{c}\right)=p_{t}(k)+\frac{1-p_{c}}{1-p_{c, \text { min }}}\left(p_{t, p c \min }-p_{t}(k)\right)
$$

It can be seen from Eqn. 6 that $p_{t}\left(k, p_{c}\right)$ depends on $p_{t}(k), k, p_{c}, p_{c, \text { min }}$, and $p_{t, p c m i n}$.

In a noiseless environment $p_{c}=1, \alpha=0$, and consequently $p_{t}\left(k, p_{c}\right)=p_{t}(k)$, i.e., $p_{t}$ is a function of $k$ only. In a noisy environment, when $p_{c}=p_{c, \min }$, then $\alpha=1$ and $p_{t}\left(k, p_{c}\right)=p_{t, p c m i n}$. If $p_{c}$ is any value 
between $p_{c, \text { min }}$ and 1 , then $p_{t}\left(k, p_{c}\right)$ varies between $p_{t}(k)$ and $p_{t, p c m i n}$ depending on $p_{c}$. According to the above discussion, $p_{t}\left(k, p_{c}\right)$ always lies between $p_{t}(k)$ and $p_{t, p c m i n}$ as shown in Figure 3.

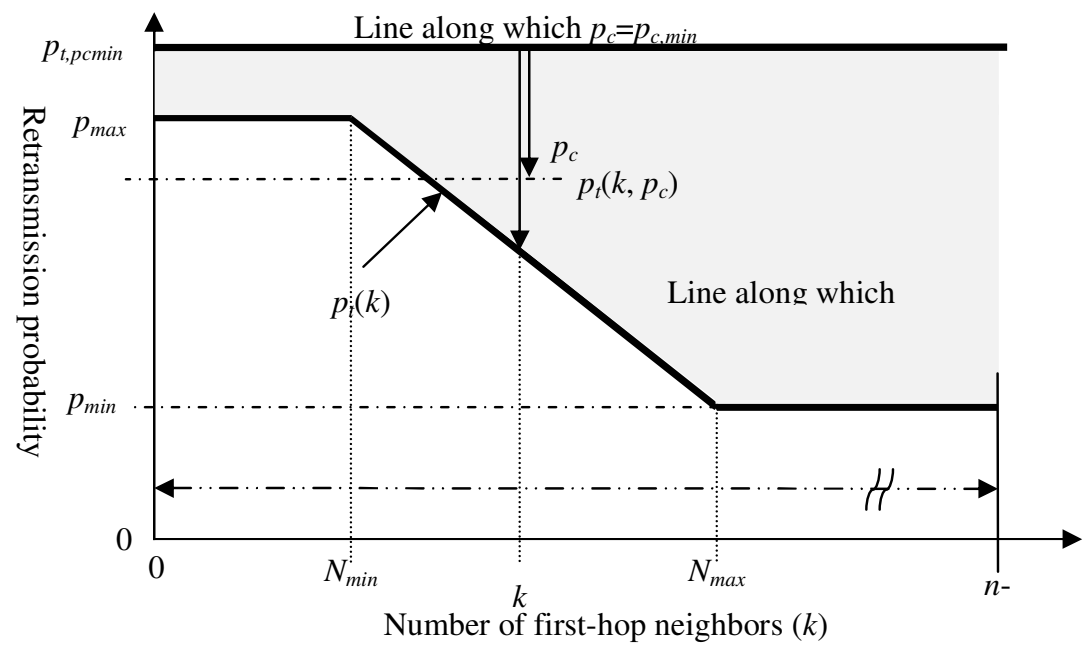

Figure 3. Variation of $p_{t}\left(k, p_{c}\right)$ (solid area) with $k$ (constant $\left.p_{t, p c m i n}\right)$

\section{ENHANCING THE DNDP AlgORITHM}

In Eqn. $5, p_{t, p c m i n}$ is assumed to be a constant value, and as it can be seen in Figure 3 that the computed $p_{t}$ is increased considerably with decreasing $p_{c}$. This may result in a significant increase in the number of retransmission, especially at high $k$. In order to alleviate the impact of this increase in $p_{t}$ and to provide adjustable measure to control that increase, we introduce the following solution. The solution is simply based on using a $k$-dependent distribution for calculating $p_{t, p c m i n}$, i.e., $p_{t, p c m i n}$ can be set as a function of $k\left(p_{t, p c m i n}(k)\right)$ as shown in Figure 4.

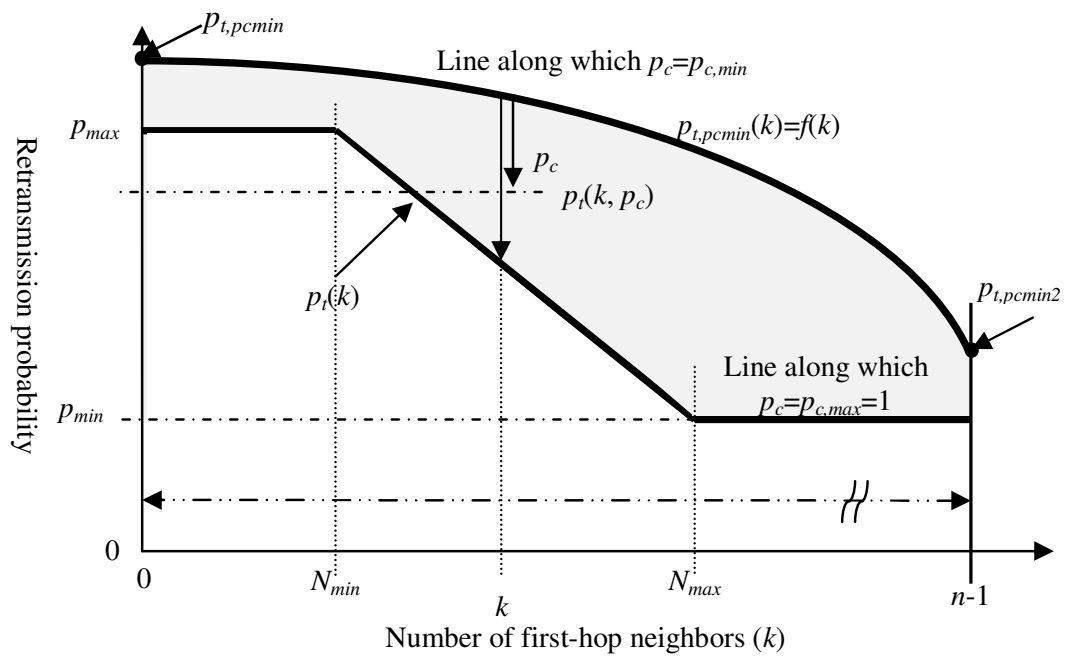

Figure 4. Variation of $p_{t}\left(k, p_{c}\right)$ (solid area) with $k\left(p_{t, p c m i n}(k)\right)$

This distribution can be discrete similar to $p_{t}(k)$ or continuous distribution, such as linear, parabolic, exponential, etc. Thus, Eqn. 6 can be re-written as: 


$$
p_{i}\left(k, p_{c}\right)=p_{i}(k)+\frac{1-p_{c}}{1-p_{c, m i n}}\left(p_{t, p e m i n}(k)-p_{i}(k)\right)
$$

Mathematical representation of the linear, parabolic, and exponential distributions of $p_{t, p c m i n}(k)$ can be given as follows:

Linear distribution:

$$
p_{t, p c \min }(k)=p_{t, p c \min 1}+\frac{p_{t, p c \min 2}-p_{t, p c \min 1}}{n-1} k
$$

Parabolic distribution:

$$
p_{t, p c \min }(k)=p_{t, p c \min 1}+\frac{p_{t, p c \min 2}-p_{t, p c \min 1}}{(n-1)^{2}} k^{2}
$$

Exponential distribution:

$$
p_{t, p c \min }(k)=p_{t, p c \min 1}+\frac{p_{t, p c \min 2}-p_{t, p c \min 1}}{1-e^{-\beta(n-1)}}\left(1-e^{-\beta k}\right)
$$

Where $p_{t, p c m i n} 1$ and $p_{t, p c m i n 2}$ are the maximum allowable $p_{t}$ in presence of noise at $k=0$ and $k=n-1$, respectively, and $\beta$ is a constant $(\beta \leq 1)$. It can be deduced from Figure 4 that due to the reduced size of the shaded area when compared with Figure $3, p_{t}\left(k, p_{c}\right)$ can be tuned in a controlled way by choosing a proper distribution for $p_{t, p c m i n}(k)$ for the sake of achieving optimum performance in terms of network reachability and number of redundant retransmissions.

\section{RESULTS AND DISCUSSION}

In this section, we investigate the effect of $p_{t, p c m i n}(k)$ on the performance of the DNDP algorithm through a number of simulations using the MANET simulator (MANSim) [18]. Also, in this section, the performance of the DNDP algorithm with different distribution for $p_{t, p c m i n}(k)$ is compared against the performance of pure flooding, fixed and dynamic probabilistic algorithms, and the DNDP algorithm with fixed $p_{t, p c m i n}$.

A number of network performance measures are calculated using MANSim, such as: network reachability $(\mathrm{RCH})$, number of retransmission (RET), average duplicate reception (ADR), average hop count (AHP), saved rebroadcast (SRB), and disconnectivity (DIS). These parameters are recommended by the Internet Engineering Task Force (IETF) group to judge the performance of the flooding optimization algorithms. Definition of these parameters can be found in [13].

This paper presents results for two parameters only, these are: RCH and RET. RCH is defined as the average number of reachable nodes by any node on the network normalized to $n$; or the

\begin{tabular}{|c|c|}
\hline \multicolumn{2}{|c|}{ Table 1 - Input parameters. } \\
\hline Parameters & Values \\
\hline Geometrical model & Random node distribution \\
\hline Network area & $600 \times 600 \mathrm{~m}$ \\
\hline Number of nodes $(n)$ & 100 nodes. \\
\hline Transmission radius $(R)$ & $100 \mathrm{~m}$ \\
\hline Average node speed $(u)$ & $5 \mathrm{~m} / \mathrm{sec}$ \\
\hline Probability of reception $\left(p_{c}\right)$ & From 0.5 to 1.0 in step of 0.1 \\
\hline Simulation time $\left(T_{\text {sim }}\right)$ & $1200 \mathrm{sec}$ \\
\hline Pause time $(\tau)$ & $\tau=0.75^{*}(R / u)=15 \mathrm{sec}$ \\
\hline Size of the mobility loop (nIntv) & $n \operatorname{Intv}=T_{\text {sim }} / \tau=80$ \\
\hline Fixed: $p_{t}$ & 0.744 \\
\hline Dynamic: $p_{\min }, p_{\max }, N_{\min }, N_{\max }, p_{1}, p_{2}$ & $0.5,0.8,4,15,0.5,0.8$ \\
\hline Fixed: $p_{t, p c \min }$ & 1 \\
\hline
\end{tabular}
probability by which a RREQ packet successfully delivered from source to destination node. RET is defined as the average number of retransmissions normalized to $n$. The input parameters for these simulations are given in Table 1. 
International Journal of Wireless \& Mobile Networks (IJWMN) Vol. 3, No. 2, April 2011

\begin{tabular}{|c|c|}
\hline Linear: $p_{t, p c \min }(k)$ & $p_{t, p c \min 1}=1$ and $p_{t, p c \min 2}=0.5$ \\
\hline Parabolic: $p_{t, p c \min }$ & $p_{t, p c \min 1}=1$ and $p_{t, p c \min 2}=0.5$ \\
\hline Exponential: $p_{t, p c \min }$ & $p_{t, p c \min l}=1$ and $p_{t, p c \min 2}=0.5, \beta=1$ \\
\hline
\end{tabular}

The main outcomes of this scenario can be summarized as follows:

- For pure and fixed/dynamic probabilistic algorithms, RCH decreases as noise-level increases, due to the high packet-loss introduced by the high noise-level; and no measure is taken by the existing probabilistic algorithms to accommodate the negative effect of increasing noise-level.

- The DNDP algorithm with any of the suggested distribution functions for $p_{t, p c m i n}(k)$ presents a better performance in terms of increasing $\mathrm{RCH}$ in presence of noise by effectively adjusting $p_{t}$ based on both $k$ and $p_{c}$. The results obtained demonstrated that the algorithm provides the highest $\mathrm{RCH}$ for various network noise-level, when compared with fixed/dynamic probabilistic algorithms.

- It can be seen from Figure 6 that the DNDP algorithm almost produces the same RCH using fixed, linear, and parabolic $p_{t, p c m i n}(k)$ distribution, while the exponential distribution produces a lower RCH, but still it is higher than what can be obtained using fixed/dynamic probabilistic algorithms. However, enhancing RCH is paid by slight increase in RET as shown in Figure 7.

- In a noiseless environment, the fixed $p_{t}$ is taken to be equal to $p_{t, a v g}$ of the dynamic approach, which is 0.744 . In a noisy environment, the average $p_{t}\left(p_{t, \text { avg }}\right)$ depends on the area under the $p_{t, p c m i n}(k)$ curve, and it can be seen in Figure 5 that the area under the curve can be arranged from the largest to the smallest as follows: fixed, parabolic, linear, and finally exponential. For the noiseless environment, $p_{t, a v g}$ for the fixed and the dynamic probabilistic algorithms are equal and always less than that for the noisy environment. This comes in line with the results presented in Figure 8.

- The dynamic probabilistic algorithm and the DNDP algorithm with any $p_{t, p c m i n}(k)$ distribution produced the same performance in noiseless environment $\left(p_{c}=1\right)$. This is because for $p_{c}=1$, the noise-correction factor $(\alpha)$ (Eqn. 5) and the noise-dependent retransmission probability $\left(p_{t}\left(p_{c}\right)\right)$ (Eqn. 3$)$ are equal to zero. Consequently $p_{t}\left(k, p_{c}\right)=p_{t}(k)$ (Eqn. 7), which means all algorithms use the same distribution for $p_{t}\left(p_{t}=p_{t}(k)\right)$.

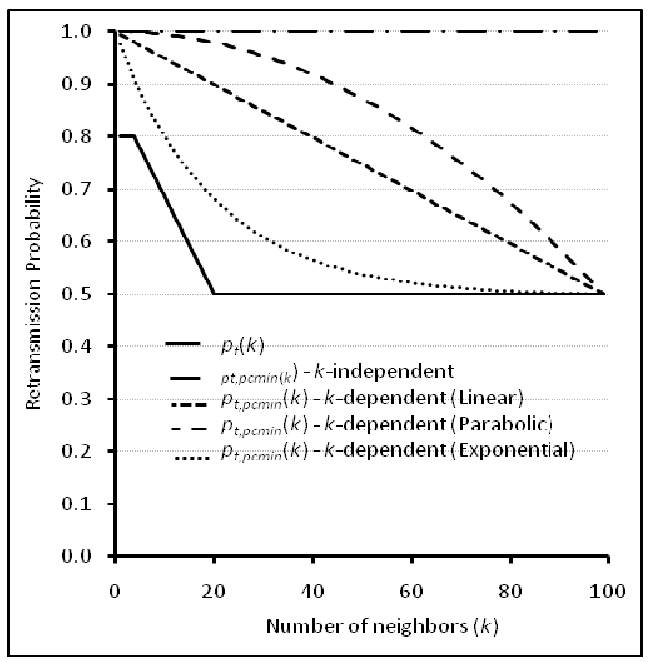

Figure 5. The variation of $p_{t, p c m i n}(k)$ with $k$

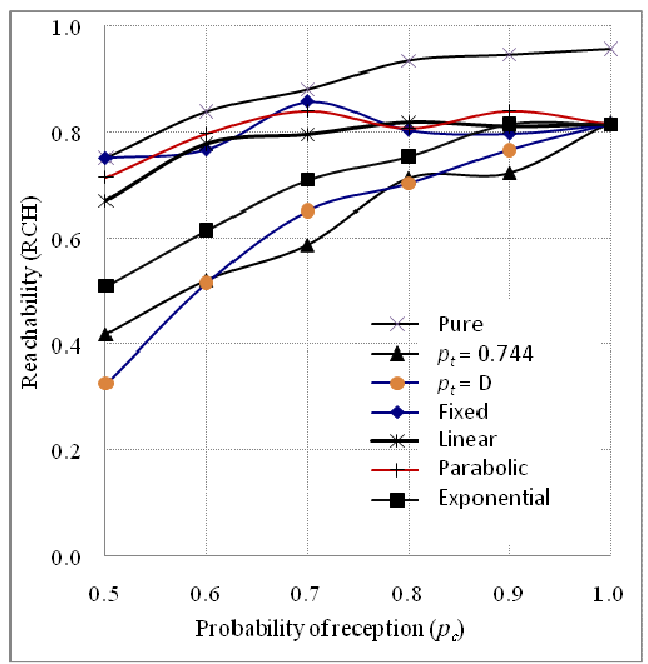

Figure 6. RCH against $p_{c}$ for various algorithms 
International Journal of Wireless \& Mobile Networks (IJWMN) Vol. 3, No. 2, April 2011

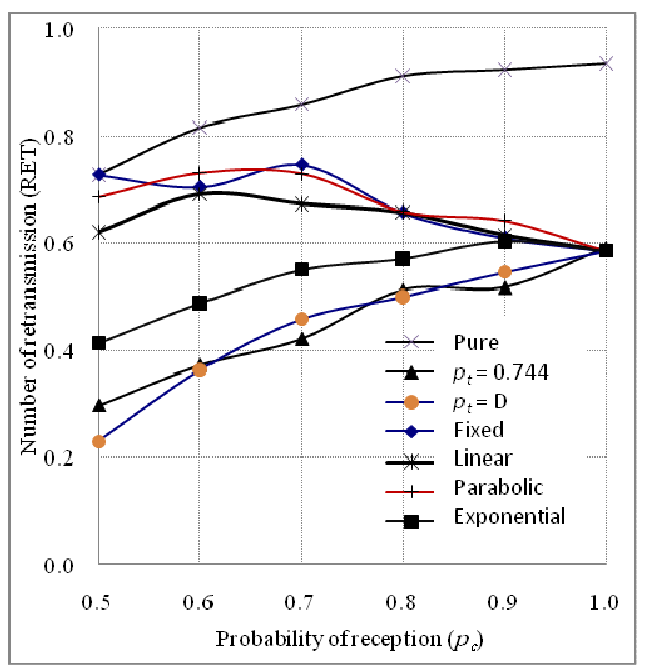

Figure 7. RET against $p_{c}$ for various algorithms.

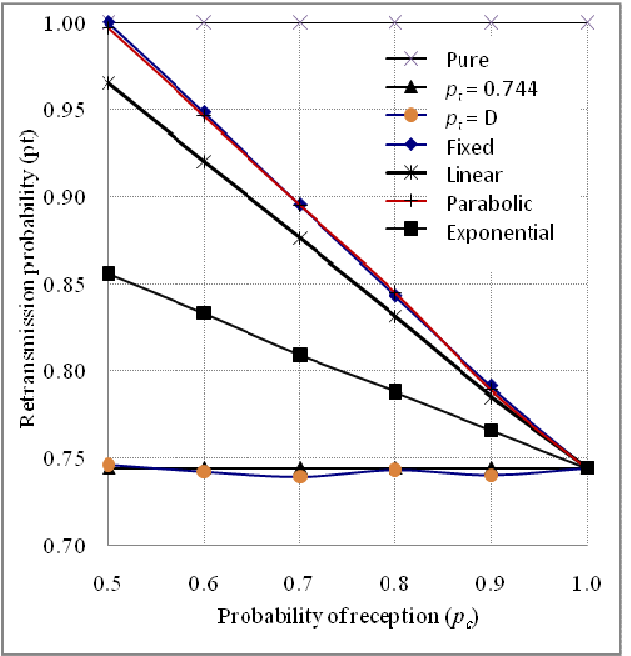

Figure 8. $p_{t, a v g}$ against $p_{c}$ for various algorithms.

\section{Conclusions}

The main conclusion of this work is that using a $k$-dependent $p_{t, p c m i n}\left(p_{t, p c m i n}(k)\right)$ enhances the performance of the DNDP algorithm, where the RCH can be improved against slight increases in RET or maintaining the same RCH against significant reduction in RET. The DNDP algorithm with linear $p_{t, p c m i n}(k)$ provides the optimum performance when compared with other distributions. The results also demonstrated that the RCH of the DNDP algorithm is close to the $\mathrm{RCH}$ of pure flooding for various network noise levels at less cost.

It is highly recommended to investigate the effect of node densities and nodes speed and other network parameters on the performance of the DNDP algorithm with various distribution for $p_{t, p c m i n}(k)$.

\section{REFERENCES}

[1] M. Bani-Yassein, M. Ould-Khaoua, L. Mackenzie, and S. Papanastasiou, "Performance Analysis of Adjusted Probabilistic Broadcasting in Mobile Ad Hoc Networks, International Journal of Wireless Information Networks, Vol. 13, No. 2, pp. 127-140, 2006.

[2] D. Scott and A. Yasinsac, "Dynamic Probabilistic Retransmission in Ad Hoc Networks", Proceedings of the International Conference on Wireless Networks (ICWN'04) (Editors: H. R. Arabnia, L. T. Yang, and C. H. Yeh), Las Vegas, Nevada, USA, CSREA Press, Vol. 1, pp. 158-164, 2004.

[3] E. Royer and C. Toh, "A Review of Current Routing Protocols for Ad Hoc Mobile Wireless Networks", IEEE Personal Communication Magazine, Vol. 6, No. 2, pp. 46-55, 1999.

[4] Z. Ismail and R. Hassan, "Evaluation of Ad Hoc on Demand Distance Vector Routing Protocol in HetMAN Architecture", Journal of Computer Science, Vol. 6, No. 7, pp. 830836, 2010.

[5] D. Johnson and D. Maltz, "Dynamic Source Routing in Ad Hoc Wireless Networks", Mobile Computing, Kluwer Academic Publishers, pp. 153-181, 1996.

[6] Z. Haas and M. Pearlman, "The Zone Routing Protocol (ZRP) for Ad Hoc Networks", Mobile Ad Hoc Network Working Group, IETF, 1998. 
International Journal of Wireless \& Mobile Networks (IJWMN) Vol. 3, No. 2, April 2011

[7] Y. Ko and N. Vaidya, "Location-Aided Routing (LAR) in Mobile Ad Hoc Networks", Journal of Wireless Networks, Vol. 6, No. 4, pp. 307-321, 2000.

[8] A. Rahman, W. Olesinski, and P. Gburzynski, "Controlled Flooding in Wireless Ad Hoc Networks. Proceedings of IEEE International Workshop on Wireless Ad Hoc Networks (IWWAN'04), University of Oulu, Finland, 2004.

[9] M. Bani-Yassein and M. Ould-Khaoua, "Applications of Probabilistic Flooding in MANETs", International Journal of Ubiquitous Computing and Communication, Vol. 1, No. 1, pp. 1-5, 2007.

[10] T. Tseng, S. Ni, Y. Chen, and J. Sheu, "The Broadcast Storm Problem in a Mobile Ad Hoc Network", Journal of Wireless Networks, Vol. 8, No. 2, pp. 153-167, 2002.

[11] H. Al-Bahadili, "Enhancing the Performance of Adjusted Probabilistic Broadcast in MANETs. The Mediterranean Journal of Computers and Networks (MEDJCN), Vol. 6, No. 4, 2010.

[12] A. Qayyum, L. Viennot, and A. Laouiti, "Multipoint Relaying for Flooding Broadcast Messages in Mobile Wireless Networks", Proceedings of the $35^{\text {th }}$ Hawaii International Conference on System Sciences (HICSS'02), Island of Hawaii, pp. 3866- 3875, 2002.

[13] H. Al-Bahadili, R. Jaradat, "Performance Evaluation of an OMPR Algorithm for Route Discovery in Noisy MANETs", International Journal of Computer Networks and Communications (IJCNC), Vol. 2, No. 1, pp. 85-96, 2010.

[14] C. Bettstetter, "The Cluster Density of a Distributed Clustering Algorithm in Ad Hoc Networks", Proceedings of the IEEE International Conference on Communications (ICC'04), Paris, France, IEEE Computer Society Press, Vol. 7, pp. 4336-4340, 2004.

[15] H. Al-Bahadili and K. Kaabneh. Analyzing the Performance of Probabilistic Algorithm in Noisy MANETs. The International Journal of Wireless \& Mobile Networks (IJWMN), Vo. 2, No. 3, pp. 83-95, 2010.

[16] H. Al-Bahadili and Y Jaradat, "Development and Performance Analysis of a Probabilistic Flooding in Noisy Mobile Ad Hoc Networks", Proceedings of the $1^{\text {st }}$ International Conference on Digital Communications and Computer Applications (DCCA'07), Irbid, Jordan, pp. 1306-1316, 2007.

[17] H. Al-Bahadili and A. Sabri, "A Novel Dynamic Noise-Dependent Probabilistic Algorithm for Route Discovery in MANETs", International Journal of Business Data Communication and Networking (IJBDCN), Vol. 7, No. 1, pp. 52-67, 2011.

[18] H. Al-Bahadili, "On the Use of Discrete-Event Simulation in Computer Networks Analysis and Design", In Handbook of Research on Discrete-Event Simulation Environments: Technologies and Applications (Eds.: E. Abu-Taieh and A. El-Sheikh,), Information Science Reference, USA, pp. 414-442, 2009.

[19] Z. J. Haas, J. Y. Halpern, and L. Li, "Gossip-Based Ad Hoc Routing", IEEE/ACM Transactions on Networking, Vol. 14, No. 3, pp. 479-491, 2006.

[20] Y. Sasson, D. Cavin, and A. Schiper, "Probabilistic Broadcast for Flooding in Wireless Mobile Ad Hoc Networks. Proceedings of IEEE Wireless Communications and Networking (WCNC'03), Vol. 2, New Orleans, LA, USA, pp. 1124-1130, 2003.

[21] J. S. Kim, Q. Zhang, and D. P. Agrawal, "Probabilistic Broadcasting Based on Coverage Area and Neighbor Confirmation in Mobile Ad Hoc Networks", Proceedings of IEEE Global Telecommunications Conference Workshops (GlobeCom'04), Dallas, Texas, USA, IEEE Computer Society Press, pp. 96-101, 2004. 
[22] C. Barrett, S. Eidenbenz, L. Kroc, M. Marathe, andL. Smith, "Parametric Probabilistic Routing in Sensor Networks", Journal of Mobile Networks and Applications, Vol. 10, No. 4, pp. 529-544, 2005.

[23] K. Viswanath, and K. Obraczka, "Modeling the Performance of Flooding in Wireless Multi-Hop Ad Hoc Networks", Journal of Computer Communications, Vol. 29, No. 8, pp. 949-956, 2005.

[24] Q. Zhang and D. P. Agrawal, "Dynamic Probabilistic Broadcasting in MANETs", Journal of Parallel and Distributed Computing, Vo. 65, No. 2, pp. 220-233, 2005.

[25] J. Abdulai, M. Ould-Khaoua, L. Mackenzie, and M. Bani-Yassin, "On the Forwarding Probability for On-Demand Probabilistic Route Discovery in MANETs", Proceedings of the $22^{\text {nd }}$ Annual UK Performance Engineering Workshop (UKPEW'06), Poole, Dorset, UK, pp. 9-15, 2006.

[26] J. Abdulai, M. Ould-Khaoua, and L. Mackenzie, "Improving Probabilistic Route Discovery in Mobile Ad Hoc Networks", Proceedings of the $32^{\text {nd }}$ Conference on Local Computer Networks (LCN '07), Dublin, Ireland, IEEE Computer Society, pp. 739-746, 2007.

[27] I. Khan, A. Javaid, and H. Qian, "Coverage-Based Dynamically Adjusted Probabilistic Forwarding for Wireless Mobile Ad Hoc Networks", Proceedings of the $1^{\text {st }}$ ACM International Workshop on Heterogeneous Sensor and Actor Networks (HeterSanet '08), Hong Kong, China, 2008, pp. 81-88, 2008.

[28] A. Hanash, A. Siddique, I. Awan, and M. Woodward, "Performance Evaluation of Dynamic Probabilistic Broadcasting for Flooding in Mobile Ad Hoc Networks", Journal of Simulation Modeling Practice and Theory, Vol. 17, No. 2, pp. 364-375, 2009.

[29] H. Al-Bahadili, "Enhancing the Performance of Adjusted Probabilistic Broadcast in MANETs", The Mediterranean Journal of Computers and Networks (MEDJCN), Vol. 6, No. 4, 2010.

\section{AUTHORS}

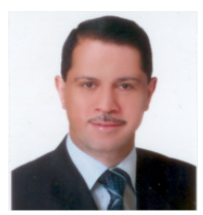

Hussein Al-Bahadili (Hbahadili@uop.edu.jo) received his B.Sc degree in Engineering from University of Baghdad, Iraq, in 1986. He received the M.Sc and PhD degree from University of London (Queen Mary College), UK, in 1988 and 1991, respectively. He is currently an associate professor at Petra University, Jordan. He is also a visiting researcher at the Centre of Wireless Networks and Communications (WNCC), University of Brunel (UK). He has published many papers in leading journals and world-level scholarly conferences. He also published a number of book chapters in high standard IT books. His research interests include computer networks design and architecture, routing protocols optimizations, parallel and distributed computing, cryptography and network security, and data compression.

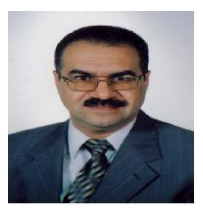

Ghassan F. Issa (gissa@uop.edu.jo) received his B.E.T degree in Electronic Engineering from the University of Toledo, Ohio, in 1983, and B.S.EE in Computer Engineering from TriState University, Indiana in 1984. He received his M.S. and Ph.D. in Computer Science from Old Dominion University, Virginia, in 1987 and 1992 respectively. He was a faculty member and department chair of Computer Science at Pennsylvania College of Technology (Penn State) from 1992-1995. He also served as faculty member and the dean of Computer Science at the Applied Science University in Amman, Jordan from 1995-2007. Currently he is an associate professor and the dean of Computer Science at Petra University, Jordan. His research interest covers block cipher, and authentication.

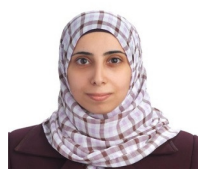

Alia Sabri (﹎.sabri@asu.edu.jo) received her B.Sc degree in Computer Science from Jordan University of Science and Technology (Jordan) in 2002. She received her M.Sc and PhD in Computer Information Systems from the Arab Academy for Banking \& Financial Sciences (Jordan) in 2005 and 2009, respectively. She is currently working as an assistance professor at the Applied Science University, Amman, Jordan. Her current research interests are in developing efficient dynamic routing protocols for mobile ad hoc networks, wireless networks management and security, and ad hoc networks modeling and simulation. 